\title{
Capítulo
} 1

\section{Utilizando Ciência de Redes no Desenvolvimento de Sistemas Complexos}

\author{
Rodrigo Pereira dos Santos, Jefferson Elbert Simões, Everton Cavalcante
}

\begin{abstract}
Resumo
Sistemas complexos são caracterizados como tal em razão dos diversos recursos envolvidos em seu ciclo de vida, bem como da interação com outros sistemas, atores e artefatos. Essas características afetam decisões de projeto, desenvolvimento, operação, governança e evolução desses sistemas, requerendo melhor compreensão dos relacionamentos entre seus elementos. Além disso, a demanda por gerir uma troca de volumes cada vez maiores de dados e a necessidade de se fazer uso eficiente de recursos computacionais e energéticos limitados são fatores críticos para esses sistemas complexos. Nessa perspectiva, este capítulo introduz como o contexto dos sistemas complexos afeta o desenvolvimento das aplicações contemporâneas e como modelos em ciência de redes podem ser utilizados para melhor compreender a estrutura $e$ características desses sistemas. De forma mais específica, por meio do estudo de duas classes de sistemas complexos (ecossistemas de software e sistemas de sistemas), este capítulo busca contribuir para: (i) compreender as características desses sistemas, que inclusive refletem-se em alguns sistemas atuais; (ii) apresentar a área de ciência de redes, em termos de modelos, ferramentas e mecanismos de análise; (iii) avaliar problemas nesse contexto, ; (iv) identificar desafios e oportunidades de pesquisa.
\end{abstract}

\begin{abstract}
Complex systems are characterized as such due to the many resources involved in their life cycle, as well as their interactions with other systems, actors, and artifacts. These characteristics affect decisions on to the design, operation, management, governance, and evolution of such systems, thus requiring a a better understanding of of the relationships among their elements. Furthermore, the demand for managing the increasing exchange of large volumes of data and the need for efficient use of limited computational and energy resources are critical factors for these complex systems. This chapter introduces how the context of complex systems affects the development of
\end{abstract}


contemporary applications and how network science models can be used to better understand the structure and characteristics of these systems. More specifically, by studying two classes of complex systems (software ecosystems and systems-of-systems), this chapter seeks to contribute to: (i) understand the characteristics of these systems, which indeed are reflected into some of today's systems; (ii) present the field of network science in terms of models, tools, and analysis mechanisms; (iii) assess problems in this context; and (iv) identify research challenges and opportunities.

\subsection{Introdução}

Sistemas complexos exercem um papel importante na vida cotidiana, ciência e economia, nos mais variados domínios de aplicação [Graciano Neto et al.2017b]. Tal complexidade advém dos diversos recursos (hardware, software, humanos, técnicos) e processos (processamento, monitoramento, gestão, comunicação etc.) envolvidos no ciclo de vida desses sistemas, bem como da interação com outros sistemas, atores e artefatos [Santos 2016]. Essas características sem dúvida afetam decisões de projeto, desenvolvimento, operação, governança e evolução, requerendo melhor compreensão dos relacionamentos entre os elementos que os compõem [Jansen et al. 2013].

Exemplos desses tipos de sistemas são os ecossistemas de software (ECOS) e os sistemas de sistemas (SoS), ambos objetos de pesquisa e desenvolvimento nos últimos anos, na academia e na indústria [Santos e Werner 2011a, Nielsen et al.2015]. Um ECOS consiste de um sistema complexo formado por atores (empresas, desenvolvedores internos e externos e usuários) e artefatos que interagem com um mercado de software cujas relações são apoiadas por uma plataforma tecnológica e realizadas pela troca de informação, recursos e artefatos (e.g., SAP, Eclipse e Android) [Jansen et al. 2009]. Por sua vez, um SoS pode ser entendido como sistema complexo que tem como constituintes outros sistemas heterogêneos e independentes, cada um deles com seu(s) próprio(s) objetivo(s) e colaborando entre si para satisfazer objetivos maiores e globais (e.g., suporte a desastres e cidades inteligentes) [Maier, 1998].

O objetivo deste capítulo é apresentar como o contexto dos sistemas complexos afeta o desenvolvimento das aplicações contemporâneas e como modelos em ciência de redes podem ser utilizados para melhor compreender a estrutura e características desses sistemas. A ciência de redes visa estudar entidades físicas e/ou virtuais e os relacionamentos entre elas utilizando teorias e métodos advindos de múltiplas áreas de conhecimento, dentre elas Matemática, Física, Computação, Estatística e Sociologia. De forma mais específica, por meio do estudo de duas classes de sistemas complexos (ECOS e SoS'), este capítulo busca contribuir para (i) compreender as características desses sistemas, que inclusive se refletem em alguns sistemas atuais, (ii) conhecer a área de ciência de redes, em termos de modelos, ferramentas e mecanismos de análise, (iii) avaliar problemas existentes nesse contexto e (iv) identificar desafios e oportunidades. A temática abordada possui significativa relação com tópicos relevantes no âmbito da comunidade da área de Sistemas de Informação, a exemplo de sistemas de sistemas de informação e complexidade de sistemas de informação.

Neste capítulo, as abreviações ECOS e SoS serão utilizadas tanto para o singular quanto para o plural: ecossistema(s) de software e sistema(s) de sistemas, respectivamente. 
Além desta introdução, este capítulo contém as seguintes seções. A Seção 1.2 discorre sobre os fundamentos acerca dos conceitos de SoS, ECOS e ciência de redes. A Seção 1.3 explora alguns exemplos de como a ciência de redes pode ser aplicada na análise de SoS e ECOS. A Seção 1.4 traz alguns desafios e oportunidades de pesquisa neste contexto. Por fim, a Seção 1.5 conclui o capítulo com as considerações finais.

\subsection{Sistemas Complexos e Ciência de Redes}

Esta seção tem por objetivo apresentar as principais características de SoS e ECOS, bem como as bases teóricas da ciência de redes, seus fenômenos e principais ferramentas de modelagem e análise.

\subsubsection{Sistemas de Sistemas (SoS)}

Tanto sistemas tradicionais quanto SoS são sistemas que integram componentes a fim de prover funcionalidades e satisfazer missões. No entanto, SoS distinguem-se de outros sistemas complexos e de larga escala devido a um conjunto de características inerentes a essa classe de sistemas, todas elas devendo ser satisfeitas para se considerar um sistema como um SoS [Maier 1998, Firesmith 2010]. Essas características, descritas brevemente adiante, são as que tornam SoS distintos dos sistemas tradicionais, requerendo assim uma mudança de paradigma no desenvolvimento desses sistemas. Com efeito, pesquisas na área já reconheceram que métodos existentes na Engenharia de Sistemas possuem aplicabilidade limitada ou não se aplicam a SoS, fazendo com que o desenvolvimento dessa classe de sistemas represente um desafio significativo [Lana et al. 2016, Azani 2019].

Independência operacional dos sistemas constituintes. Sistemas constituintes em um SoS são operacionalmente independentes no sentido de que proveem suas próprias funcionalidades e satisfazem suas próprias missões mesmo quando não estão em cooperação com outros sistemas no escopo do SoS. Essa característica não se observa em sistemas tradicionais, uma vez que seus componentes constituintes operam conforme foram designados para prover suas funcionalidades.

Independência gerencial dos sistemas constituintes. Sistemas constituintes que participam de um SoS são frequentemente desenvolvidos por diferentes organizações, com seus próprios stakeholders, equipes de desenvolvimento, processos e recursos, além de apresentarem um ciclo de vida próprio e terem autonomia para gerenciar seus próprios recursos. Esse não é o caso de sistemas tradicionais, em que seus componentes constituintes estão atrelados a ele e todas as decisões são feitas sobre o sistema, de maneira centralizada.

Distribuição geográfica dos sistemas constituintes. Esta característica refere-se à dispersão dos sistemas constituintes, sendo necessária alguma forma de conectividade para permitir comunicação e troca de informações [Nielsen et al. 2015]. Assim, sistemas constituintes de um SoS estão fisicamente desacoplados e trocam apenas informações entre si.

Desenvolvimento evolucionário do SoS. Um SoS pode evoluir constantemente em resposta a mudanças, sejam estas relacionadas ao ambiente, aos seus sistemas constituintes, às suas funcionalidades ou às suas missões. Devido a sua independência gerencial, os sistemas constituintes podem evoluir por conta própria sem o controle do 
SoS, que, por sua vez, deve absorver tais mudanças e minimizar possíveis efeitos colaterais indesejáveis.

Comportamentos emergentes no SoS. Em sistemas tradicionais, comportamentos e interações dos seus componentes constituintes são em sua grande maioria previsíveis e controláveis da perspectiva do sistema. No entanto, mesmo que os sistemas constituintes de um SoS sejam previsíveis, suas independências operacional e gerencial fazem com que o comportamento do SoS emerja em tempo de execução. Isso se coloca como um desafio significativo ao desenvolvimento dessa classe de sistemas, uma vez que SoS dependem fortemente de comportamentos emergentes para satisfazer suas missões e prover novas funcionalidades [Inocêncio et al. 2019]. Tais funcionalidades resultam da colaboração entre os sistemas constituintes e não podem ser providas por nenhum deles se considerados de forma isolada.

Por fim, uma característica adicional de SoS é a sua inerente dinamicidade, que faz com que o sistema esteja constantemente sujeito a mudanças, isto é, seus sistemas constituintes podem ser integrados, operados e reconfigurados em tempo de execução, na maioria das vezes de forma não planejada. Os sistemas constituintes concretos a integrarem um SoS em tempo de execução frequentemente são conhecidos de forma parcial ou mesmo desconhecidos quando do projeto desse SoS. Com isso, os sistemas constituintes devem ser descobertos, selecionados e integrados em tempo de execução para que sejam identificados os arranjos adequados desses sistemas que contribuem para alcançar os objetivos gerais do SoS com base em suas capacidades [Gomes et al. 2015].

\subsubsection{Ecossistemas de Software (ECOS)}

Para apoiar a globalização da indústria de software, avanços na criação de métodos para desenvolver sistemas de grande porte, de longo prazo, interconectados e sujeitos a rápida velocidade de implantação e evolução têm sido empreendidos [Bosch 2016]. Esse cenário requer profissionais com a habilidade de abstrair a complexidade do sistema como um todo e entender que um sistema é resultado da combinação entre software, hardware e peopleware, constituída sobre uma plataforma comum [Messerschmitt e Szyperski 2003, Santos et al. 2012], levando aos ECOS. Grandes empresas como Amazon, Apple, Google, Microsoft e SAP lideram o desenvolvimento de ECOS, o que contribuiu para a crescente pesquisa no tema [Manikas e Hansen 2013, Manikas 2016].

No mercado de software atual, empresas não trabalham mais como unidades independentes ou entregam produtos distintos, mais sim dependem de outros fornecedores de componentes e de infraestruturas vitais, tais como sistemas operacionais, bibliotecas, lojas de componentes e plataformas [Bosch e Olsson 2018]. Nesse sentido, ECOS traz uma visão holística, uma vez que a participação de atores externos, sejam eles conhecidos ou não, passou a fazer parte da rotina das empresas, gerando uma nova maneira de estruturar modelos de negócio e de desenvolvimento de software [Yang et al. 2017]. Os estudos relativos a esse contexto visam ainda explicar novos elementos que estão atrelados ao software. Por exemplo, ao invés de gerir a variabilidade dos produtos e serviços de software oferecidos aos seus clientes, as empresas passam a ter de prover formas para que eles customizem as suas próprias soluções segundo a necessidade/contexto (e.g., ECOS móveis) [Fontão et al. 2017].

Entre as definições de ECOS, há três principais. Jansen et al. (2009) definem ECOS como um conjunto de atores funcionando como uma unidade que interage com um 
mercado distribuído entre software e serviços, juntamente com as suas relações frequentemente apoiadas por uma plataforma tecnológica ou por um mercado comum e realizadas pela troca de informação, recursos e artefatos. Bosch (2009) define ECOS como um conjunto de soluções de software que possibilitam, apoiam e automatizam as atividades e transações feitas por atores em um ecossistema social ou de negócios e por organizações que provêm soluções. Lungu et al. (2010) definem um ECOS como uma coleção de projetos de software que são desenvolvidos e evoluem em conjunto em um mesmo ambiente.

Para van den Berk et al. (2010), um ECOS possui basicamente três elementos: centralizador $(h u b)$, plataforma (tecnologia ou mercado) e agentes de nicho (niche players). Nesse contexto, o centralizador é o dono da plataforma e os agentes de nicho são os envolvidos que podem utilizá-la para gerar valor para si e para o ecossistema. Por exemplo, no caso do ECOS Windows, a Microsoft é o centralizador, o Windows é a plataforma e as demais empresas, desenvolvedores externos e usuários são os agentes de nicho, que usam a plataforma para construir/usar suas aplicações. Um problema que aparece neste contexto é a existência de muitas empresas e relacionamentos, que envolvem fornecedores, desenvolvedores externos e usuários, o que pode dificultar decisões no desenvolvimento da plataforma do ECOS [Serebrenik e Mens 2015].

Um dos benefícios para uma empresa ou desenvolvedor se tornar um membro de um ECOS está na oportunidade de explorar a inovação aberta para que atores colaborem a fim de alcançar benefícios regionais e globais. O esforço dos atores externos em desenvolver para uma plataforma de ECOS pode transformar a inovação em algo positivo para si e seus clientes, contribuindo também para o centralizador por estender/melhorar a plataforma e engajar mais envolvidos [Fontão et al. 2018]. A proximidade entre o centralizador e os atores externos é fundamental para o sucesso de um empreendimento de software em ECOS. Por outro lado, entre as dificuldades enfrentadas neste contexto, estão: (i) estabelecer e modelar relacionamentos entre os atores de um ECOS; (ii) definir estratégias de negócio; (iii) manter arquitetura da plataforma; (iv) monitorar a evolução do ECOS; (v) romper obstáculos de comunicação de requisitos em projetos distribuídos geograficamente, e; (vi) construir ferramentas para acelerar a interação social e melhorar o processo de tomada de decisão [Barbosa et al.2013].

Algumas classificações foram desenvolvidas pela comunidade acadêmica visando facilitar a compreensão do conceito de ECOS a partir da modelagem e análise de seus elementos constituintes. Uma estratégia para se modelar e analisar ECOS foi introduzida por Campbell e Ahmed (2010) e explorada em detalhes por Santos e Werner (2011a, 2011b, 2012a, 2012b). Os autores apresentam uma visão de ECOS em três dimensões que visam entender as funções assumidas por cada um dos pilares de um ECOS (Figura 1.1):

- Arquitetura (ou dimensão técnica): envolve a plataforma (infraestrutura com tecnologias e aplicações) na qual o ecossistema vai estar inserido e explora questões da arquitetura de software, linha de produto e processos;

- Negócio (ou dimensão transacional): envolve conhecimento do mercado, de decisões que os atores devem tomar sobre modelos de negócio, de definição do portfólio de produtos do ECOS, de estratégias de licenças e de vendas;

- Social: envolve explorar como a rede de atores se relaciona dentro do ECOS para atingir os seus objetivos, bem como fomentar o crescimento do ECOS por meio de proposições de valor em que todos possam ser beneficiados. 


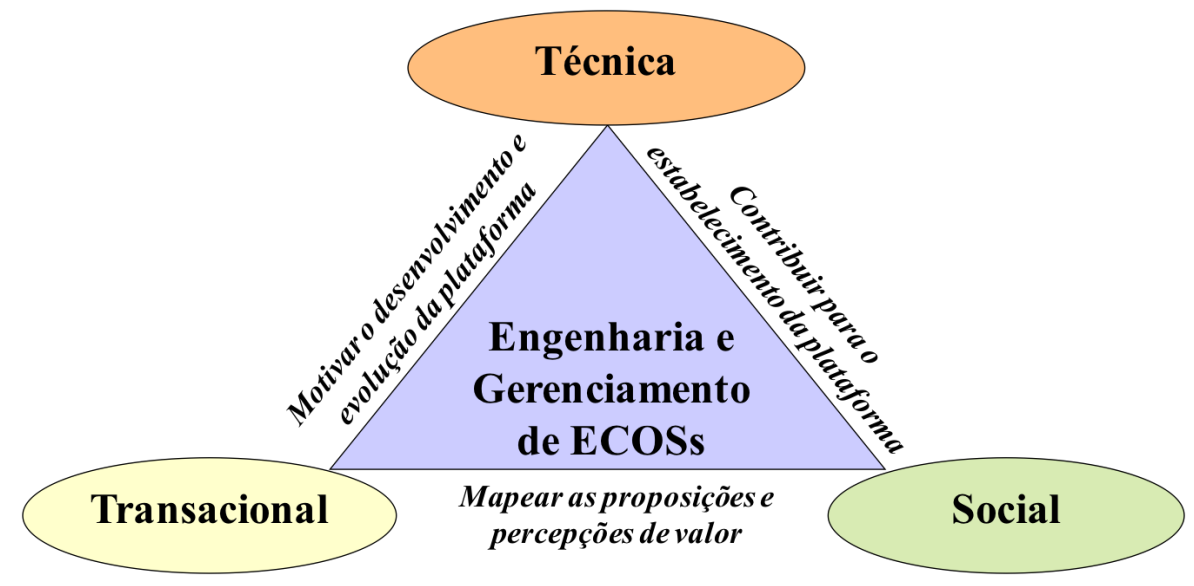

Figura 1.1: Visão de ECOS em três dimensões.

Fonte: Santos e Werner (2011a, 2011b, 2012a, 2012b)

Vale ressaltar ainda que há um número significativo de estudos que investigam ECOS centrados em plataformas do tipo free open source software (FOSS), seja pela disponibilidade de dados ou pela importância da colaboração entre os atores como elemento fortalecedor da plataforma [Franco-Bedoya et al. 2017]. Além disso, existem ECOS centrados em uma plataforma de ativos de software, representando uma linha de produtos de software [McGregor 2012], ou ainda voltados para um mercado de software e serviços de Tecnologia da Informação, formando um ecossistema de negócio para processos de inovação (aberta ou fechada). Alguns elementos comumente modelados e analisados em um ECOS são: (i) a arquitetura da plataforma, em termos de estabilidade, gerência de evolução, extensibilidade, segurança e confiabilidade [Amorim et al.2016]; (ii) processos de negociação de requisitos, sendo necessário alinhar necessidades reais com soluções, componentes e portfólios [Fotrousi et al. 2015]; (iii) desenvolvimento colaborativo [van der Maas 2016]; (iv) modelos e métricas para redes de produção de software [Santos e Oliveira 2013], e; (v) modelos de negócio, como o de ECOS formado por organizações de pequeno e médio porte [Valença et al. 2018].

Dado que o contexto de ECOS reúne um conjunto de problemas em aberto para os diferentes domínios de sistemas, modelos e análises de casos reais têm utilizado recursos como UML, $i^{*}$ e grafos [Coutinho et al. 2017]. É fundamental assim modelar elementos de arquitetura de modo que esta deva ser estendida para tratar gerenciamento, regras de negócio e restrições, integração e existência de múltiplas funcionalidades, seja para a plataforma central ou para as aplicações construídas sobre ela. Segurança e confiabilidade são atributos de qualidade críticos para equilibrar modularidade e flexibilidade na especificação da arquitetura da plataforma, focando em interoperabilidade e manutenção de interfaces de programação [Motta et al.2017]. Além disso, inconsistências geradas por evoluções devem ser verificadas/validadas por meio das dependências de componentes e serviços da plataforma, o que requer mecanismos de coordenação [Graciano Neto et al. 2017a]. A constante evolução no contexto de um ECOS demanda processos adaptáveis para que o desenvolvimento tenha interoperabilidade, implantação e liberação de versões estáveis da plataforma. Como consequência, a análise e o projeto arquitetural passam a enfatizar estratégias para identificação de metas de negócio e de requisitos emergentes, além de táticas para 
avaliação arquitetural. O processo de elicitação de requisitos torna-se um desafio, dado que novos atores se juntam àqueles comumente envolvidos no processo de desenvolvimento de software, criando um corpo numeroso e distribuído no ECOS [Silva et al. 2017]. Por fim, cadeias de valor e de resultados passam a apoiar a engenharia de requisitos [Yu e Deng 2011], além da modelagem, análise e visualização de redes sociais, técnicas e sociotécnicas para tratar redes de influência e interoperabilidade.

\subsubsection{Ciência de Redes}

Nos anos 1930, Jacob Levy Moreno apresentou uma série de representações de redes sociais, hoje conhecidas como sociogramas. Nessas representações, Moreno captura as relações sociais entre alunas de diversas turmas da New York Training School for Girls através de diagramas [Moreno 1934], como mostra a Figura 1.2. Nascia aqui a disciplina de análise de redes sociais, um ramo da Sociologia que visa realizar estudos quantitativos sobre redes sociais tendo como informação de base o padrão de conexões entre as pessoas que compõem tais redes.

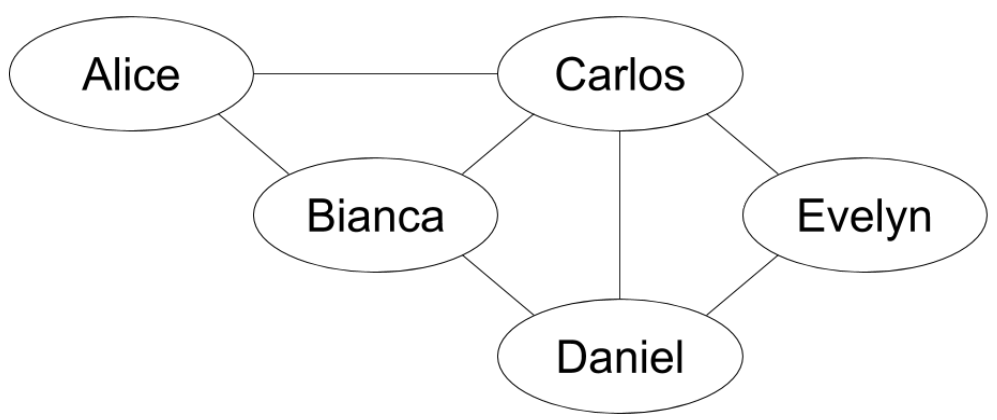

Figura 1.2: Exemplo de sociograma representando os relacionamentos de amizade entre cinco pessoas que constituem uma rede social. Neste exemplo, a rede apresentada possui sete relacionamentos no total.

Sociogramas implicitamente compreendem as relações sociais representadas como relações par-a-par, isto é, relações cuja existência ou ausência é observada entre pares de pessoas ${ }^{2}$. Assim como redes sociais, muitos outros sistemas podem ser intuitivamente vistos como uma combinação complexa de relacionamentos par-a-par. Um sistema de transporte metroviário pode ser visto como um conjunto de estações conectadas sucessivamente pelas linhas de metrô que as atravessam. Uma teia alimentar é constituída de relações predatórias entre as inúmeras espécies que habitam uma comunidade ecológica. A infraestrutura da Internet é formada por diversas organizações chamadas sistemas autônomos, que constituem uma infraestrutura própria de roteadores e negociam conexões com outros sistemas autônomos para troca de tráfego. Até mesmo o sistema nervoso humano, formado por bilhões de neurônios, pode ser interpretado como uma rede de conexões entre esses neurônios através das quais impulsos elétricos se propagam. Em todos esses contextos, um sistema real é modelado através da representação de um conjunto de objetos ligados uns aos outros por relacionamentos para-par de alguma natureza. Essa abstração é chamada de rede.

${ }^{2}$ Em alguns contextos, relações podem envolver mais do que duas partes, a exemplo de um contrato de aluguel envolvendo o inquilino, o proprietário e a imobiliária ou a transferência de um jogador de futebol de uma equipe para outra. Estes dois exemplos constituem relações ternárias. 
Redes não são uma abstração nova em diversas áreas de conhecimento, em particular nas áreas de Matemática e Computação, nas quais são mais conhecidas por grafos. De fato, desde os primeiros resultados de teoria de grafos há quase 300 anos, esse objeto matemático se tornou um dos mais estudados em ambas as áreas. Para diversas comunidades acadêmicas que utilizam resultados derivados de teoria de grafos, os termos grafo e rede são utilizados de forma quase intercambiável, com leves diferenças de significado. Em ciência de redes, em geral utiliza-se o termo rede em referência à representação de objetos e relacionamentos observados no mundo real (rede social, rede metroviária, rede neuronal), enquanto o uso do termo grafo geralmente tem a conotação de que a rede representada não é relevante para a discussão em questão. Essa convenção será seguida neste texto.

A área de ciência de redes tem como objetivo central analisar e entender as redes que surgem nos mais diversos domínios de conhecimento, os processos que elas afetam e os processos que são afetados por elas. Naturalmente, a ciência de redes é fortemente interdisciplinar, pois converge conhecimentos, técnicas e objetivos de áreas como Matemática, Computação, Física, Estatística, Biologia, Economia e Sociologia. Além disso, sendo uma área de conhecimento com delimitação relativamente recente (cerca de 20 anos) e fortemente motivada pelo surgimento de grandes massas de dados extraídas dos mais diversos contextos, a ciência de redes também se apresenta como marcadamente empírica e quantitativa.

Em termos de terminologia, os objetos de uma rede são frequentemente chamados de vértices ou nós e as conexões/relacionamentos entre eles são ditos arestas ou elos. Dois vértices conectados diretamente por uma aresta são ditos vizinhos. De maneira geral, tanto os vértices quanto as arestas podem conter rótulos e/ou atributos que codificam alguma informação extraída da realidade. No caso das arestas, quanto um atributo identifica a intensidade do relacionamento codificado, tal atributo é denominado peso da aresta.

Inúmeras métricas topológicas já foram propostas e utilizadas para quantificar propriedades de redes e de seus vértices e arestas. A mais fundamental delas é o grau de um vértice, definido como a quantidade de vizinhos que esse vértice possui. O grau é o primeiro indicador do quão bem-conectado um vértice está em uma rede e, por isso, muitos resultados em teoria de grafos são obtidos em função dos graus mínimo ou máximo de uma rede. Apesar disso, o grau de um vértice ainda é um pedaço de informação muito simples para informar algo sobre aquele vértice individual e ainda menos relevante no contexto da rede como um todo. Em vez disso, interessa-se na frequência com que cada grau ocorre na rede, isto é, a distribuição de grau.

A representação mais simples e direta da distribuição de grau é um histograma indicando a quantidade de vértices com cada grau, mas em alguns casos é interessante também observar variantes em sua versão cumulativa (isto é, mostrando a quantidade de vértices com grau menor ou igual ao valor dado) ou cumulativa complementar (quantidade de vértices com grau maior ou igual). Também é frequente normalizar o gráfico pela quantidade total de vértices na rede, de forma que o valor plotado seja, na verdade, a proporção de vértices em vez do número absoluto. Um exemplo é apresentado na Figura 1.3.

A distribuição de grau é uma propriedade fundamental na caracterização da estrutura de uma rede. Em particular, como ilustrado na Figura 1.4, muitas redes reais 
apresentam distribuição de grau do tipo "causa pesada", caracterizada pela ocorrência de vértices, em proporção não-desprezível, com grau ordens de grandeza maior que a média. Isto indica uma forte desigualdade nos graus dos vértices, com número de outliers de grau extremamente alto concentrando a maior parte das arestas. Barabási e Albert (1999) mostraram que esse tipo de distribuição surge naturalmente de redes resultantes de um processo de crescimento, no qual novos vértices vão sendo gradualmente adicionados à rede, acoplado a um mecanismo de anexação preferencial, que confere a vértices com grau mais alto algum nível de favorecimento ao receber novas arestas.

\section{Distribuição de grau}

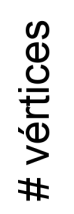

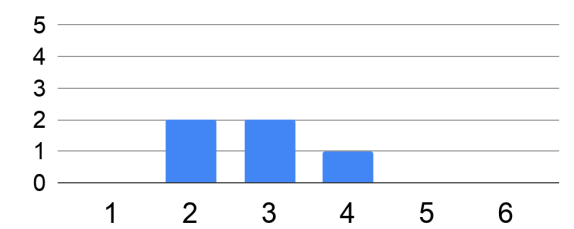

Grau

\section{Distribuição de grau}
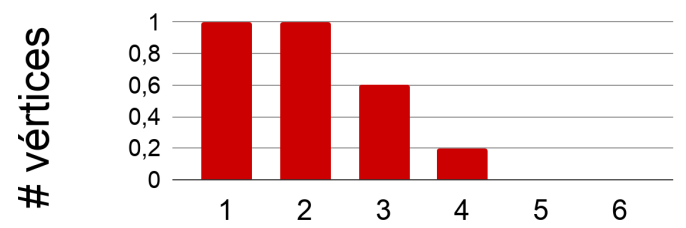

Grau

Figura 1.3: Distribuição de grau da rede apresentada na Figura 1.2. 0 histograma à esquerda apresenta a distribuição de grau em sua forma mais simples, em que cada barra indica a quantidade de vértices com 0 valor de grau correspondente. 0 histograma à direita apresenta a distribuição de grau cumulativa complementar: cada barra indica a quantidade de vértices (normalizada entre 0 e 1) com grau menor ou igual ao valor correspondente.
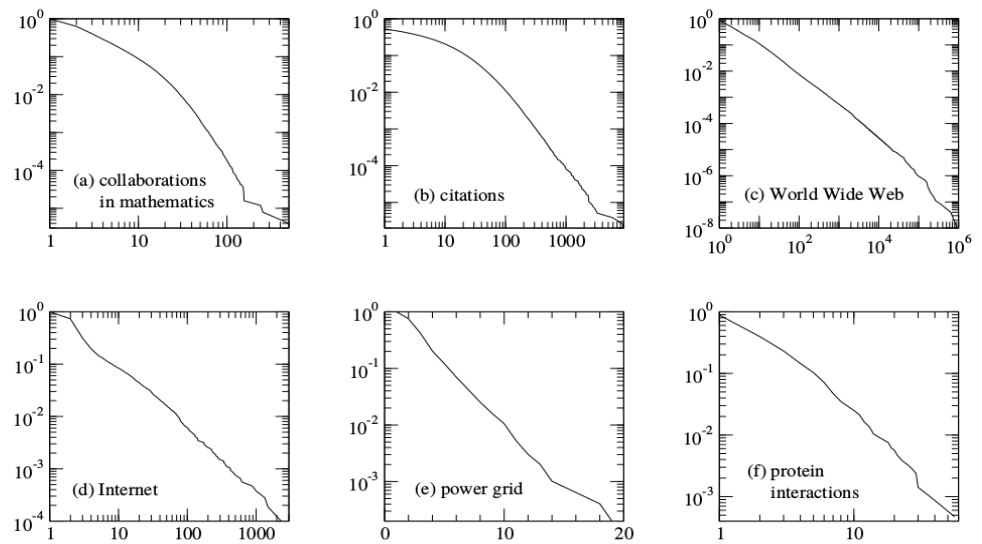

Figura 1.4: Distribuição de grau, na forma cumulativa complementar, de redes diversas: (a) rede de colaborações entre matemáticos; (b) rede de citações entre artigos científicos; (c) rede de páginas Web interligadas por hiperlinks; (d) infraestrutura da Internet; (e) malha elétrica; (f) rede de interações entre proteínas. Em todos os gráficos, exceto o (e), os eixos estão em escala logarítmica e a forma do gráfico é aproximadamente uma linha reta, o que sugere uma distribuição de grau de causa pesada, em particular do tipo lei de potência [Newman 2003].

Outra forma de analisar a estrutura de uma rede é observar as distâncias entre os vértices. Dados dois vértices quaisquer na rede, a distância entre eles é o comprimento do menor caminho que sai de um vértice e chega no outro, onde todos os passos desse caminho obrigatoriamente precisam seguir as arestas da rede. Um exemplo de fenômeno que pode ser estudado utilizando esta métrica é o chamado "efeito mundo pequeno": a existência de caminhos extremamente curtos entre quaisquer dois vértices de redes muito 
grandes. Por exemplo, uma análise da rede de amizades no Facebook, realizada em 2011, descobriu que, em média, 4,32 passos conectam dois usuários americanos aleatoriamente selecionados, número que aumenta para apenas 4,74 passos para dois usuários aleatórios no mundo todo [Backstrom et al. 2012]. Esse resultado parece contra-intuitivo, considerando que a quantidade média de amigos de uma pessoa qualquer no Facebook (o grau deste vértice) é muito menor do que a quantidade total de usuários cadastrados. $\mathrm{O}$ efeito mundo pequeno, observado experimentalmente em diversas redes sociais ao longo de várias décadas [Travers e Milgram 1969, Dodds et al. 2003, Backstrom et al. 2012], se tornou famoso na literatura de ciências sociais sob o nome de "seis graus de separação" e inspirou o número de Erdös ${ }^{3}$, parte canônica do folclore matemático, e jogos como o "Six Degrees of Kevin Bacon".

Conectado a este fenômeno, tem-se uma métrica fundamental, a clusterização. Intuitivamente, a clusterização indica uma tendência de dois vértices que possuam um vizinho comum serem, eles próprios, vizinhos também (intuitivamente, "os amigos dos meus amigos são meus amigos também"). Essa tendência é intuitivamente observada em diversas classes de rede, como observado, por exemplo, pela teoria de equilíbrio em ciências sociais. Quantitativamente, a clusterização é obtida normalizando a quantidade de triângulos em uma rede, isto é, a quantidade de trios de vértices dois-a-dois conectados uns aos outros. Algumas classes de redes, como as redes tecnológicas (e.g., malhas elétricas), apresentam clusterização próxima de zero enquanto outras, como as redes sociais, apresentam clusterização relativamente alta. Essa propriedade é, à primeira vista, conflitante com o efeito mundo pequeno, em redes com grau médio baixo. Isto porque, quanto mais arestas em uma rede têm o papel de conectar vértices a distâncias baixas, conferindo à rede um alto índice de clusterização, menos arestas estarão disponíveis para conectar esses vértices ao restante da rede e diminuir a distância média na rede como um todo. Propostas de resolução desta contradição foram feitas, por exemplo, em contextos como Sociologia [Granovetter 1973] e Neurologia [Gallos et al. 2012], com o modelo "mundo pequeno" de Watts e Strogatz (1998) ganhando expressiva notoriedade.

Para compreender o papel de vértices específicos em uma rede qualquer, uma classe extremamente importante de métricas são as medidas de centralidade. Na literatura de ciência de redes, o termo centralidade indica uma forma quantitativa de mensurar a importância de um determinado vértice em comparação com os demais, com vértices mais importantes recebendo um valor maior de centralidade. Naturalmente, existem diversas formas de conceber, em termos qualitativos, a importância de um vértice qualquer, dependendo da natureza da rede e também da aplicação em questão. Em razão disso, já foram propostas na literatura diversas medidas de centralidade. Algumas das mais importantes são listadas abaixo:

- Centralidade de intermediação (betweenness centrality): obtida, para um dado vértice da rede, contando quantos caminhos mínimos na rede (caminhos que ligam algum par de vértices da forma mais curta possível) passam por este vértice; um vértice com alta centralidade de intermediação é importante, pois serve como ponte para conectar uma quantidade muito grande de outros vértices e sua remoção da rede tende a causar o maior impacto em termos de aumento das distâncias entre os demais vértices;

\footnotetext{
${ }^{3}$ The Erdös Number Project: https://oakland .edu/enp/

${ }^{4}$ The Oracle of Bacon: http://oracleofbacon.org
} 
- Centralidade de proximidade (closeness centrality): obtida, para um dado vértice da rede, somando as distâncias deste vértice para todos os outros e tomando o inverso desta soma; um vértice com alta centralidade de proximidade é importante, pois está, em média, mais perto dos outros vértices da rede em comparação com os demais;

- Centralidade de autovetor: formulação recursiva de centralidade que determina que um vértice é tão central quanto mais centrais forem seus vizinhos; nesta formulação, a centralidade de um vértice é proporcional à soma das centralidades de seus vizinhos, mas o valor específico atribuído a cada vértice é dependente de um parâmetro de normalização, com o vértice mais central da rede geralmente recebendo valor de centralidade igual a 1 ;

- Centralidade de PageRank: variante da centralidade de autovetor na qual a centralidade de um vértice depende da centralidade de seus vizinhos por um fator inversamente proporcional ao grau de cada um deles; a ideia por trás desta métrica de centralidade é a base do algoritmo de ranqueamento de páginas Web inovador apresentado pelo Google em 1998 e utilizado em seu motor de busca até hoje.

Por fim, é importante também mencionar as métricas de assortatividade. Estas métricas exigem que cada vértice da rede possua um atributo associado, que pode ser endógeno (calculado a partir da estrutura da rede, como o grau de cada vértice) ou exógeno à rede (obtido externamente, como a idade em uma rede social ou a quantidade de páginas dos artigos em uma rede de citações). Diz-se que uma rede apresenta homofilia com relação a um atributo se há uma tendência das arestas de uma rede a conectarem vértices com valores iguais ou próximos deste atributo, enquanto a tendência inversa, com arestas conectando vértices com valores distintos, corresponde a um cenário de heterofilia.

Por exemplo, a análise de uma rede de blogs políticos nos Estados Unidos, ilustrada na Figura 1.5 (na qual arestas indicam hiperlinks entre os blogs), identificou homofilia para o atributo exógeno "orientação política" [Interian e Ribeiro 2018]. Em contraste, redes tecnológicas como a Internet possuem uma estrutura fortemente hierárquica na qual vértices com grau alto tendem a se conectar a vértices com grau baixo, apresentando, assim, heterofilia com relação ao atributo endógeno "grau" [Newman 2003]. Métricas diferentes são utilizadas dependendo, por exemplo, se o atributo em questão é categórico, numérico discreto ou contínuo. Por outro lado, a correspondência entre os fenômenos de homofilia e heterofilia e valores específicos da métrica de assortatividade depende também da distribuição empírica do atributo na rede.

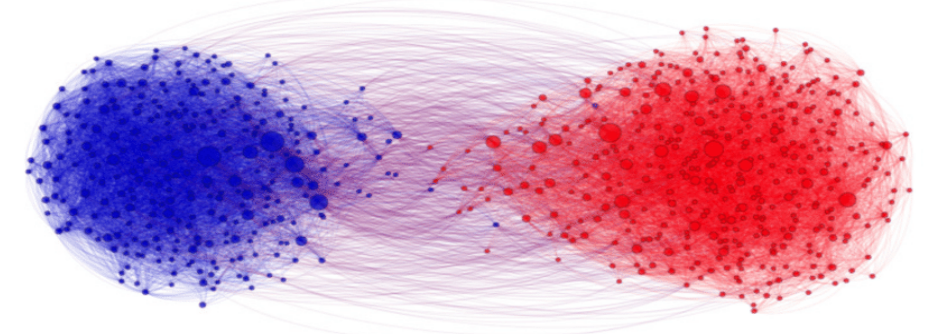

Figura 1.5: Rede de blogs políticos nos Estados Unidos. $O$ agregado à esquerda corresponde a alinhamento com o Partido Democrata, enquanto o agregado à direita corresponde a alinhamento com o Partido Republicano [Interian e Ribeiro 2018]. 


\subsection{Aplicando Ciência de Redes na Análise de SoS e ECOS}

Ciência de redes, nas palavras de Barabási (2016), é "uma plataforma capacitante, fornecendo novas ferramentas e perspectiva para uma vasta gama de problemas científicos, de redes sociais a projeto de remédios". Em troca, ela exige que seja incorporada uma nova forma de pensar e enxergar a complexidade dos sistemas. É preciso compreender que "por trás de cada sistema complexo existe uma rede intrincada que codifica as interações entre os componentes do sistema" [Barabási 2016]. Esses componentes podem ser elementos computacionais, pessoas, partes de um organismo, países ou até mesmo segmentos de informação.

Dessa forma, em uma tentativa de linearizar o processo científico utilizando as ferramentas que ciência de redes oferece, pode-se afirmar que o primeiro passo consiste na identificação das redes relacionadas ao problema em mãos. Mais precisamente, devese identificar quais são os componentes do sistema e qual é a natureza do relacionamento entre esses componentes, os quais corresponderão, respectivamente, a vértices e arestas da rede. Este passo é fundamental, pois ele não apenas define quais dados deve-se obter para construir uma imagem da rede que possa futuramente ser quantitativamente analisada, mas também define a semântica subjacente a todas as métricas que serão computadas a partir destes dados.

É importante ressaltar que, de forma geral, existem diversos elementos de um sistema complexo que podem ser compreendidos como componentes interagentes, de forma que existem múltiplas redes que podem ser identificadas em um dado contexto. Por exemplo, ao estudar a dinâmica de produção científica, uma análise do ponto de vista do conhecimento produzido leva naturalmente à construção da rede de citações, na qual os vértices são artigos científicos e as arestas correspondem a citações entre eles, enquanto o emprego de uma abordagem sociológica torna mais interessante o estudo da rede de colaborações, em que os vértices são pesquisadores e as arestas são induzidas pela publicação de trabalhos em coautoria.

Além disso, a granularidade com que os dados podem ser obtidos também influencia a definição da rede. Por exemplo, a rede neuronal de organismos simples pode ser analisada a nível de neurônios (a rede neural do verme Caenorhabditis elegans foi totalmente mapeada em 1986 [White et al. 1986]), enquanto para organismos mais complexos como o ser humano é necessário construir a rede a nível de áreas funcionais. O estudo da Internet, uma rede de roteadores interligados por enlaces de comunicação, raramente desfruta de informação suficiente sobre enlaces específicos e a análise topológica da Internet geralmente é feita sobre a rede de sistemas autônomos (regiões topológicas da Internet sob administração única).

Seguem alguns exemplos de redes que podem naturalmente ser identificadas nos contextos de ECOS e SoS:

- um SoS pode ser diretamente modelado como uma rede de sistemas constituintes interagentes, em que os sistemas correspondem aos vértices da rede e as trocas de mensagens entre eles são representadas pelas arestas;

- em ECOS, uma plataforma de desenvolvimento e compartilhamento de artefatos de software induz naturalmente uma dinâmica de coutilização em projetos, que pode ser analisada através de uma rede de coutilização: os artefatos constituem os 
vértices da rede e as arestas correspondem à utilização simultânea em um projeto, com um peso indicando a frequência de coutilização;

- tanto em ECOS quanto em SoS, qualquer software desenvolvido de forma modular pode ser visto como uma rede de dependências, com os vértices correspondendo aos módulos e as dependências diretas entre eles induzindo as arestas;

- tanto em ECOS quanto em SoS, a dinâmica de desenvolvimento de artefatos de software envolve interações entre desenvolvedores, o que torna interessante a análise da rede de colaborações entre desenvolvedores: os vértices são os desenvolvedores e as arestas indicam a quantidade de projetos em que eles colaboraram ou artefatos que eles produziram em conjunto.

O passo seguinte consiste em identificar métricas que capturem propriedades da rede relacionadas com o problema a ser analisado. Naturalmente é interessante utilizar métricas já propostas na literatura e com validade comprovada pela comunidade científica, mas, em muitos casos, as métricas existentes podem ser insuficientes para capturar o problema atacado de maneira significativa. Neste caso, é interessante que novas métricas, adequadas para o caso em questão, sejam propostas. Destaca-se que a discussão de como as características de ECOS e SoS anteriormente apresentadas podem ser analisadas por meio da ciência de redes, a fim de mostrar como seus modelos apoiam decisões de desenvolvimento, tem sido pouco relatada em artigos científicos publicados sobre sistemas complexos.

Mesmo as métricas mais básicas de ciência de redes podem ser interessantes para uma análise preliminar da rede em questão. Seguem alguns exemplos utilizando as métricas apresentadas na Seção 1.2:

1) Na rede de interações de um SoS, é interessante que nenhum sistema concentre uma grande parte das interações, evitando assim a formação de gargalos ou pontos únicos de falha. Essa hipótese pode ser verificada calculando a distribuição de grau desta rede e verificando se é observada uma distribuição bastante concentrada. A existência de um conjunto de vértices com grau muito maior do que a média sugere que tais vértices podem constituir gargalos e pontos de falha do SoS. Em outras palavras, a distribuição de grau da rede de interações serve de indicativo para a robustez do SoS como um todo;

2) Na rede de dependências de um software, como cada módulo idealmente possui um objetivo relativamente pontual, em que ele dever-se-ia relacionar com um conjunto limitado de outros módulos. Novamente, essa hipótese pode ser verificada calculando a distribuição de grau desta rede. Uma distribuição de grau muito desigual pode ser um indício de que um determinado módulo está concentrando um número excessivo de funções, evidenciando uma modularização inadequada;

3) A rede de colaborações de desenvolvedores codifica os padrões de interação existentes entre as pessoas envolvidas nos processos de desenvolvimento. Considere-se um cenário em que se gostaria de compor um comitê consultivo técnico para a análise de novos projetos a serem adicionados a uma plataforma, como ocorre em um ECOS. Um critério interessante para a seleção de pessoas para comporem esse comitê seria encontrar desenvolvedores que mais colaboram nesta comunidade e, com isso, conhecem melhor os critérios que constituem um 
bom projeto de software. Esse critério sugere que se procure os desenvolvedores que possuem valor alto em alguma métrica de centralidade, como, por exemplo a centralidade de autovetor;

4) Considere-se ainda uma plataforma de desenvolvimento de software em que cada artefato possui um conjunto de rótulos (tags), indicando de maneira resumida seu contexto e seu propósito. Na rede de coutilização de artefatos desta plataforma, é esperado que artefatos semelhantes não sejam utilizados simultaneamente por não agregarem muita funcionalidade ao software já desenvolvido, como acontece na plataforma de um ECOS. Isso sugere que um grau alto de homofilia nesta rede é um indicador de redundância no conjunto de artefatos existentes, ou um sinal de que muitos artefatos só funcionam em conjunto e talvez devessem ser distribuídos como um único artefato.

É importante ter em mente que, quantitativamente falando, é natural que redes de naturezas diferentes apresentem propriedades topológicas diferentes. Por exemplo, é razoável que uma rede de colaborações de um ECOS, sendo uma rede social, apresente um índice alto de clusterização, ao contrário de uma rede tecnológica como a rede de interações em um SoS. Por isso, é interessante fornecer um benchmark para comparar as métricas extraídas das redes reais. Esse benchmark é tradicionalmente dado por um modelo estocástico para redes, do qual pode-se extrair um valor esperado para tais métricas analiticamente ou através de simulações computacionais. Inúmeros modelos já foram propostos na literatura, sendo alguns dos mais conhecidos o modelo Erdős-Rényi, o modelo de blocos estocástico, o modelo Barabási-Albert e o modelo Watts-Strogatz. Cada modelo é mais adequado para representar redes obtidas por diferentes processos de formação e, em casos específicos, também pode ser interessante propor um modelo adequado para a rede em questão.

\subsection{Desafios e Oportunidades de Pesquisa}

Como desdobramentos, sugere-se investigar alguns desafios e oportunidades de pesquisa no campo de ciência de redes para sistemas complexos como ECOS e SoS. Primeiramente, é importante investigar como a diversidade de tecnologias, sistemas, organizações e atores afetam o desenvolvimento de sistemas de informação modernos, dado que diferentes redes sociotécnicas são constituídas de maneira dinâmica e, para tanto, requerem técnicas e ferramentas para modelagem e análise que considerem essa característica dos tipos de sistemas complexos discutidos neste capítulo.

Outra linha de investigação refere-se a como decisões de projeto, desenvolvimento, operação, governança e evolução desses sistemas podem ser apoiadas por técnicas que combinem mineração de dados e visualização de informação, considerando os vários níveis de abstração, os diferentes pontos de vista dos stakeholders e as carências de uma gestão de conhecimento orgânica e institucionalizada. Mais ainda, é importante verificar como identificar, gerir e avaliar a demandas e necessidades de diferentes stakeholders em um cenário cuja troca de volumes cada vez maiores de dados e a necessidade de se fazer uso eficiente de recursos computacionais e energéticos limitados têm sido fatores críticos, dado que sistemas complexos possuem, em sua essência, comportamento emergente e dinamismo em constituir e desfazer determinadas configurações para cumprir missões, como em SoS. 
Outro aspecto interessante a investigar é como apoiar uma melhor compreensão dos relacionamentos entre os elementos envolvidos em sistemas complexos, uma vez que se torna importante não apenas entender que elementos compõem as redes sociotécnicas, mas também os diversos tipos de relacionamentos que podem ocorrer entre tais elementos. Um primeiro passo pode ser partir de tipos reportados em mapeamentos sistemáticos e explorar técnicas da ciência de redes para sumarização de informação.

Por fim, mas não menos importante, estudos experimentais tais como estudos de caso de natureza interpretativa e qualitativa podem explorar informações de contexto e contribuir para uma base de conhecimento que possa guiar diferentes stakeholders em seus processos de tomada de decisão. Essa é uma questão relevante uma vez que não é uma tarefa fácil prover soluções passíveis de generalização para problemas enfrentados na gestão e engenharia desses sistemas, mas cujas experiências de diferentes cenários ajudam a gerar insights.

\subsection{Considerações Finais}

Este capítulo introduziu como o contexto dos sistemas complexos vem afetando o desenvolvimento das aplicações contemporâneas e como modelos em ciência de redes podem ser utilizados para melhor compreender a estrutura e características desses sistemas. De forma mais específica, por meio do estudo de duas classes de sistemas complexos (ECOS e SoS), buscou-se compreender as características desses sistemas (que inclusive refletem-se em alguns sistemas atuais), conhecer a área de ciência de redes (alguns modelos, ferramentas e mecanismos de análise), avaliar problemas e identificar desafios e oportunidades de pesquisa. Destaca-se que, apesar de ECOS e SoS serem sistemas que materializam o desafio de se tratar a complexidade com uma visão mais sistêmica, poucos trabalhos têm reportado a aplicação de técnicas e modelos de ciência de redes neste contexto, o que reforça o desafio para a pesquisa e prática. Isso evidencia desafios e abre oportunidades de pesquisa para a comunidade de sistemas de informação, uma vez que requer uma visão integrada de pessoas, processos e tecnologia.

\section{Referências}

Amorim, S. S., McGregor, J. D., Almeida, E. S., Chavez, C. F. G. (2017) "Software ecosystems' architectural health: Another view", Proceedings of the Joint 5th International Workshop on Software Engineering for Systems-of-Systems and 11th Workshop on Distributed Software Development, Software Ecosystems and Systemsof-Systems. USA, IEEE, pp. 66-69.

Azani, C. (2009) "An open systems approach to System of Systems Engineering". In: Jamshidi, M., ed. Systems of Systems Engineering: Innovations for the 21st Century. USA, John Wiley \& Sons, Inc., pp. 21-43.

Backstrom, L., Boldi, P., Rosa, M., Ugander, J., Vigna, S. (2012) "Four degrees of separation", Proceedings of the 4th Annual ACM Web Science Conference. USA, ACM, pp. 33-42.

Barabási, A.-L. (2016) "Network Science". United Kingdom, Cambridge University Press.

Barabási, A.-L., Albert, R. (1999) "Emergence of scaling in random networks". Science, vol. 286, no. 5439, pp. 509-512. 
Barbosa, O., Santos, R. P., Alves, C., Werner, C., Jansen, S. (2013) "A systematic mapping study on software ecosystems from a three-dimensional perspective". In: Jansen, S., Brinkkemper, S., Cusumano, M. A. eds. Software ecosystems: Analyzing and managing business networks in the software industry. United Kingdom/USA, Edward Elgar Publishing, pp. 59-81.

Bosch, J. (2009) "From software product lines to software ecosystem", Proceedings of 13th International Software Product Line Conference. USA, ACM, pp. 1-10.

Bosch, J. (2016) "Speed, data, and ecosystems: The future of Software Engineering", IEEE Software, vol. 33, no. 1, pp. 82-88.

Bosch, J., Olsson, H. H. (2018) "Ecosystem traps and where to find them", Journal of Software: Evolution and Process, vol. 30, no. 11.

Campbell, P. R. J., Ahmed, F. (2010) "A three-dimensional view of Software Ecosystems", Proceedings of the 4th European Conference on Software Architecture: Companion Volume. USA, ACM, pp. 81-84.

Coutinho, E. F., Viana, D., Santos, R. P. (2017) "An Exploratory Study on the need for modeling software ecosystems: The case of SOLAR SECO", Proceedings of the 9th International Workshop on Modelling in Software Engineering. USA, IEEE, pp. 4753.

Dodds, P. S., Muhamad, R., Watts, D. J. (2003) "An experimental study of search in global social networks", Science, vol. 301, no. 5634, pp. 827-829.

Firesmith, D. (2010) "Profiling systems using the defining characteristics of systems of systems (SoS)", Technical report. USA, Software Engineering Institute, Carnegie Mellon University.

Fontão, A., Ábia, B., Wiese, I., Estácio, B., Quinta, M., Santos, R. P., Dias-Neto, A. C. (2018) "Supporting governance of mobile application developers from mining and analyzing technical questions in stack overflow", Journal of Software Engineering Research \& Development, vol. 6.

Fontão, A., Dias-Neto, A. C., Viana, D. (2017) "Investigating factors that influence developers' experience in mobile software ecosystems", Proceedings of the Joint 5th International Workshop on Software Engineering for Systems-of-Systems and 11th Workshop on Distributed Software Development, Software Ecosystems and Systemsof-Systems. USA, IEEE, pp. 55-58.

Franco-Bedoya, O., Ameller, D., Costal, D., Franch, X. (2017) "Open source software ecosystems: A systematic mapping", Information and Software Technology, vol. 91, pp. 160-185.

Gallos, L. K., Makse, H. A., Sigman, M. (2012) "A small world of weak ties provides optimal global integration of self-similar modules in functional brain networks", Proceedings of the National Academy of Sciences, vol. 109, no. 8, pp. 2825-2830.

Gomes, P., Cavalcante, E., Maia, P., Batista, T., Oliveira, K. (2015) "A systematic mapping on discovery and composition mechanisms for systems-of-systems", Proceedings of the 41st Euromicro Conference on Software Engineering and Advanced Applications. USA, IEEE, pp. 191-198. 
Graciano Neto, V. V., Cavalcante, E., El Hachem, J., Santos, D. S. (2017a) "On the interplay of business process modeling and missions in systems- of-information systems", Proceedings of the Joint 5th International Workshop on Software Engineering for Systems-of-Systems and 11th Workshop on Distributed Software Development, Software Ecosystems and Systems-of-Systems. USA, IEEE, pp. 72-73.

Graciano Neto, V. V., Santos, R. P., Araujo, R. M., (2017b) "New challenges in the social Web: Towards systems-of-information systems ecosystems". In: Anais do VIII Workshop sobre Aspectos da Interação Humano-Computador na Web Social. CEUR Workshop Proceedings, vol. 2039, pp. 1-12.

Granovetter, M. S. (1973) "The strength of weak ties", American Journal of Sociology, vol. 78, no. 6, pp. 1360-1380.

Inocêncio, T. J., Gonzales, G. R., Cavalcante, E., Horita, F. E. A. (2019) "Emergent behavior in systems-of-systems: A systematic mapping study", Proceedings of the XXXIII Brazilian Symposium on Software Engineering. USA, ACM, pp. 140-149.

Interian, R., Ribeiro, C. C. (2018) "An empirical investigation of network polarization", Applied Mathematics and Computation, vol. 339, pp. 651-662.

Jansen, S., Brinkkemper, S., and Cusumano, M. A. (2013) "Software ecosystems: Analyzing and managing business networks in the software industry". United Kingdom/USA, Edward Elgar Publishing.

Jansen, S., Finkelstein, A., Brinkkemper, S. (2009) "A sense of community: A research agenda for software ecosystems", Proceedings of the 31st International Conference on Software Engineering. USA, ACM, pp. 187-190.

Lana, C. A., Souza, N. M., Delamaro, M. E., Nakagawa, E. Y., Oquendo, F., Maldonado, J. C. (2016) "Systems-of-systems development: Initiatives, trends, and challenges", Proceedings of the XLII Latin American Computing Conference. USA, IEEE, pp. 585596.

Lungu, M., Lanza, M., Girba, T., Robbes, R. (2010) "The Small Project Observatory: Visualizing software ecosystems", Science of Computer Programming, vol. 75, no. 4, pp. 264-275.

Maier, M. W. (1998) "Architecting principles for systems-of-systems", Systems Engineering, vol. 1, no. 4, pp. 267-284.

Manikas, K. (2016) "Revisiting software ecosystems research: A longitudinal literature study", Journal of Systems and Software, vol. 117, pp. 84-103.

Manikas, K., Hansen, K. M. (2013) "Software ecosystems - A systematic literature review", Journal of Systems and Software, vol. 86, no. 5, pp. 1294-1306.

McGregor, J. D. (2012) "Ecosystem modeling and analysis", Proceedings of 16th International Software Product Line Conference - Volume 2. USA, ACM, p. 268.

Messerschmitt, D. G., Szyperski, C. (2003) "Software ecosystem: Understanding an Indispensable technology and industry". USA, MIT Press.

Moreno, J. L. (1934) "Who shall survive?: A new approach to the problem of human interrelations". Nervous and Mental Disease Publishing Co. 
Motta, R. C., Oliveira, K. M., Travassos, G. H. (2017) "Rethinking interoperability in contemporary software systems", Proceedings of the Joint 5th International Workshop on Software Engineering for Systems-of-Systems and 11th Workshop on Distributed Software Development, Software Ecosystems and Systems-of-Systems. USA, IEEE, pp. 9-15.

Newman, M. J. (2003) "The Structure and Function of Complex Networks", SIAM Review, vol. 45, no. 2, pp. 167-256.

Nielsen, C. B., Larsen, P. G., Fitzgerald, J., Woodcock, J., Peleska, J. (2015) "Systems of Systems Engineering: Basic concepts, model-based techniques, and research directions", ACM Computing Surveys, vol. 48, no. 2.

Santos, R. P. (2016) "Managing and monitoring software ecosystem to support demand and solution analysis", PhD Thesis. Brazil, COPPE/UFRJ.

Santos, R. P., Oliveira, J. (2013) "Análise e aplicações de redes sociais em ecossistemas de software", Anais do IX Simpósio Brasileiro de Sistemas de Informação. Brasil, SBC, pp. 19-24.

Santos, R. P., Werner, C. M. L. (2011a) "A proposal for Software Ecosystems Engineering". In: Proceedings of the 3rd International Workshop on Software Ecosystems. CEUR Workshop Proceedings, vol. 746, pp. 40-51.

Santos, R. P., Werner, C. (2011b) "Treating business dimension in software ecosystems", Proceedings of the 3rd ACM/IFIP International Conference on Management of Emergent Digital EcoSystems. USA, ACM, pp. 197-201.

Santos, R. P., Werner, C. M. L. (2012a) "Treating social dimension in software ecosystems through ReuseECOS Approach", Proceedings of the 6th International Conference on Digital Ecosystem Technologies - Complex Environment Engineering. USA, IEEE, pp. 1-6.

Santos, R. P., Werner, C. (2012b) "ReuseECOS: An approach to support global software development through software ecosystems", Proceedings of the 7th IEEE International Conference on Global Software Engineering Workshops. USA, IEEE, pp. 60-65.

Santos, R. P., Werner, C., Barbosa, O., Alves, C. (2012) "Software ecosystems: Trends and impacts on Software Engineering", Proceedings of the 26th Brazilian Symposium on Software Engineering. USA, ACM, pp. 206-210.

Serebrenik, A., Mens, T. (2015) "Challenges in software ecosystems research", Proceedings of the 2015 European Conference on Software Architecture Workshops. USA, ACM.

Silva, R. T., Aguiar, L. G. F., Audacio, E. D., Genvigir, E. C. (2017) "Identifying actors to support software ecosystem health", Proceedings of the Joint 5th International Workshop on Software Engineering for Systems-of-Systems and 11th Workshop on Distributed Software Development, Software Ecosystems and Systems-of-Systems. USA, IEEE, pp. 76-77.

Travers, J., Milgram, S. (1969) "An experimental study of the small world problem", Sociometry, vol. 32, no. 4, pp. 425-443. 
Valença, G., Alves, C., Jansen, S. (2018) "Strategies for managing power relationships in software ecosystems", Journal of Systems and Software, vol. 144, pp. 478-500.

van den Berk, I., Jansen, S., Luinenburg, L. (2010) "Software ecosystems: A software ecosystem strategy assessment model", Proceedings of the 4th European Conference on Software Architecture: Companion Volume. USA, ACM, pp. 135-142.

van der Maas, J. (2016) "Evolution of collaboration in open source software ecosystems", Master Thesis. Netherlands, Utrecht University.

Watts, D. J., Strogatz, S. H. (1998) "Collective dynamics of 'small-world' networks", Nature, vol. 393, pp. 440-442.

White, J. G., Southgate, E., Thomson, J. N., Brenner, S. (1986). "The structure of the nervous system of the nematode Caenorhabditis elegans", Philosophical Transactions of the Royal Society of London B Biological Sciences, vol. 314, no. 1165, pp. 1-340.

Yang, Z., Jansen, S., Gao, X., Zhang, D. (2016) "On the future of solution composition in software ecosystems". In: Bañares J., Tserpes K., Altmann J., eds. Proceedings of the 13th International Conference on Economics of Grids, Clouds, Systems, and Services. Lecture Notes in Computer Science, vol 10382. Switzerland, Springer International Publishing AG.

Yu, E., Deng, S. (2011) "Understanding software ecosystems: A strategic modeling approach". In: Proceedings of the 3rd International Workshop on Software Ecosystems. CEUR Workshop Proceedings, vol. 746, pp. 65-76.

\section{Sobre os autores}

Rodrigo Santos é Professor Adjunto do Departamento de Informática Aplicada (DIA) e membro efetivo do Programa de Pós-Graduação em Informática (PPGI) da Universidade Federal do Estado do Rio de Janeiro (UNIRIO), do qual foi Coordenador do Curso de Mestrado (2019-2020). Lidera o Laboratório de Engenharia de Sistemas Complexos da UNIRIO (LabESC). Doutor e Mestre em Engenharia de Sistemas e Computação pela COPPE/UFRJ, onde realizou também o seu Pós-Doutorado (2016), e Bacharel em Ciência da Computação pela UFLA. Atuou como pesquisador visitante na University College London (2014-2015). É editor-chefe da iSys: Revista Brasileira de Sistemas de Informação e organizou edições especiais em periódicos como iSys, JBCS e JISA, além de volume na série Springer CCIS. É membro da Sociedade Brasileira de Computação (SBC) desde 2006, atuando como Coordenador do Comitê Gestor da Comissão Especial de Sistemas de Informação (CE-SI), membro do Comitê Gestor da Comissão Especial de Jogos e Entretenimento Digital (CE-Jogos) e membro efetivo do Grupo de Interesse em Educação em Computação (GIEC). Tem experiência na área de Ciência da Computação, com ênfase em Engenharia de Software e Sistemas de Informação. Seus principais campos de atuação são Engenharia de Sistemas Complexos (especialmente ecossistemas de software e sistemas de sistemas) e Educação em Engenharia de Software. Foi coordenador científico de mais de vinte eventos (simpósios, trilhas e workshops) no Brasil e no exterior e proferiu comunicações (palestras, minicursos e tutoriais) em mais de vinte eventos nacionais. Publicou mais de 150 artigos em periódicos e congressos, sendo alguns deles premiados, e recebeu distinções acadêmicas como revisor de destaque em conferências e como orientador de trabalhos em concursos de teses e dissertações da SBC. Link para Currículo Lattes: http://lattes .cnpq.br/8613736894676086. 
Jefferson Elbert Simões é Professor Adjunto do Departamento de Informática Aplicada (DIA) da Universidade Federal do Estado do Rio de Janeiro (UNIRIO). É Mestre e Doutor em Engenharia de Sistemas e Computação pela COPPE/Universidade Federal do Rio de Janeiro (UFRJ), com período de Doutorado Sanduíche na École Polytechnique Fédérale de Lausanne (EPFL), e Engenheiro de Computação e Informação Magna cum Laude pela UFRJ (2010). Possui interesse em ciência de redes, modelos probabilísticos, educação à distância, sistemas de computação, algoritmos e grafos. Link para Currículo Lattes: http://lattes.cnpq.br/6938694286834426.

Everton Cavalcante é Professor Adjunto do Departamento de Informática e Matemática Aplicada (DIMAp) da Universidade Federal do Rio Grande do Norte (UFRN), Natal, Brasil. Possui dupla diplomação de Doutor em Ciência da Computação pela Universidade Federal do Rio Grande do Norte e Docteur en Sciences et Technologies de l'Information et de la Communication pela Université Bretagne Sud, França (2016). Possui experiência na área de Ciência da Computação com ênfase em arquitetura de software e sistemas distribuídos, atuando principalmente nos seguintes temas: middleware, Computação em Nuvem, Computação Ubíqua, Internet das Coisas, cidades inteligentes, reconfiguração dinâmica de software, linguagens de descrição arquitetural e sistemas de sistemas. É docente permanente do Programa de Pós-Graduação em Sistemas e Computação (PPgSC) da UFRN e integra o Núcleo Integrador de Pesquisa e Inovação em Engenharia de Software (SETe) do Instituto Metrópole Digital (IMD) da UFRN, onde é coordenador do curso de Bacharelado em Engenharia de Software e vice-coordenador no Programa de Residência em Tecnologia da Informação. Link para Currículo Lattes: http://lattes.cnpq.br/5065548216266121. 